\title{
UCRL-PROC-201748
}

LAWRENCE LIVERMORE NATIONAL LABORATORY

\section{Probing Chiral Interactions in Light Nuclei}

P. Navratil

Janaury 15, 2004

$17^{\text {th }}$ International IUPAP Conference on Few-Body Problems in Physics

Durham, NC

June 5-10, 2003 


\section{Disclaimer}

This document was prepared as an account of work sponsored by an agency of the United States Government. Neither the United States Government nor the University of California nor any of their employees, makes any warranty, express or implied, or assumes any legal liability or responsibility for the accuracy, completeness, or usefulness of any information, apparatus, product, or process disclosed, or represents that its use would not infringe privately owned rights. Reference herein to any specific commercial product, process, or service by trade name, trademark, manufacturer, or otherwise, does not necessarily constitute or imply its endorsement, recommendation, or favoring by the United States Government or the University of California. The views and opinions of authors expressed herein do not necessarily state or reflect those of the United States Government or the University of California, and shall not be used for advertising or product endorsement purposes. 


\section{Probing chiral interactions in light nuclei}

A. Nogga ${ }^{\mathrm{a}},{ }^{*}$ E. Epelbaum ${ }^{\mathrm{b}}$, P. Navrátil ${ }^{c}$, W. Glöckle ${ }^{\mathrm{b}}$, H. Kamada ${ }^{\mathrm{d}}$, Ulf-G. Meißner ${ }^{\mathrm{e}}$, H. Witała ${ }^{f}$, B.R. Barrett ${ }^{\mathrm{a}}$, J.P. Vary ${ }^{\mathrm{g}}$

${ }^{a}$ University of Arizona, Tucson, AZ, USA

${ }^{\mathrm{b}}$ Ruhr-Universität Bochum, Bochum, Germany

${ }^{\mathrm{c}}$ Lawrence Livermore National Laboratory, Livermore, California, USA

${ }^{\mathrm{d}}$ Kyushu Institute of Technology, Kitakyushu, Japan

'Universität Bonn, Helmholtz Institut für Strahlen- und Kernphysik(Theorie), Bonn, Germany

${ }_{\mathrm{f}}^{\mathrm{J} J a g i e l l o n i a n}$ University, Cracow, Poland

${ }^{8}$ Iowa State University, Ames, Iowa, USA

Chiral two- and three-nucleon interactions are studied in few-nucleon systems. We investigate the cut-off dependence and convergence with respect to the chiral expansion. It is pointed out that the spectra of light nuclei are sensitive to the three-nucleon force structure. As an example, we present calculations of the $1^{+}$and $3^{+}$states of ${ }^{6} \mathrm{Li}$ using the no-core shell model approach. The results show contributions of the next-to-next-to leading order terms to the spectra, which are not correlated to the three-nucleon binding energy prediction.

\section{INTRODUCTION}

The application of chiral perturbation theory to nuclear systems promises a much better understanding of nuclear forces. Its application has recently been extended to $3 \mathrm{~N}$ and $4 \mathrm{~N}$ systems [1-3]. This development is complicated by the fact, that the subleading $2 \pi$ exchange too strongly contributes to the two-nucleon $(2 \mathrm{~N})$ force at short distances, which leads to spurious bound states. This is a major obstacle for few-nucleon (FN) calculations. A new approach for the regularization will solve this problem [4].

Parallel to this development, we would like to study the convergence properties of chiral interactions in FN systems. As motivated in Ref. [2], we cure the strong attraction by reducing the strength of the subleading $2 \pi$ exchange. This leads to a good agreement with the $2 \mathrm{~N}$ data and removes the spurious bound states. Therefore, the chiral interaction based on this approach is an ideal model to investigate the convergence of the chiral expansion

*present address: Institute for Nuclear Theory, University of Washington, Seattle, WA 98195-1550 
in FN systems. We also stress that we maintain the consistency of $2 \mathrm{~N}$ and three-nucleon $(3 \mathrm{~N})$ forces, because the same strength constants enter in the $3 \mathrm{~N}$ force $(3 \mathrm{NF})$.

In this work, we consider two ways to get insight into the size of higher order effects. Firstly, we compare the results of predictions at NLO to predictions at NNLO, and, secondly, we study the dependence on the cut-off value $\Lambda$, which determines at which momentum scale the Lippmann-Schwinger equation is truncated for the regularization. The dependence of FN observables on the cut-off should in magnitude be comparable to higher order contributions, because it finally must be absorbed into higher order contact interactions. Force models for both orders and depending on the cut-off have been developed in Refs. [5,2].

One important aspect of this systematic approach to nuclear interactions is that it results in consistent $2 \mathrm{~N}$ and $3 \mathrm{~N}$ forces. At NLO no $3 \mathrm{NF}$ appears, whereas at NNLO, additionally to the $2 \pi$ exchange $3 \mathrm{NF}$, two more structures show up, which involve two unknown low-energy constants (LEC's) [6,3]. Because of these free parameters it is easy to adjust the forces to predict the experimental $3 \mathrm{~N}$ binding energy (BE). The four-nucleon $(4 \mathrm{~N}) \mathrm{BE}$ is not expected to be sensitive to the structure of the forces, because it is strongly correlated to the $3 \mathrm{~N} \mathrm{BE} \mathrm{[7].} \mathrm{On} \mathrm{the} \mathrm{other} \mathrm{side} \mathrm{it} \mathrm{is} \mathrm{known} \mathrm{that} \mathrm{the} \mathrm{spectra} \mathrm{and} \mathrm{BE's}$ of light nuclei depend on the structure of the 3NF [8,9]. Therefore, they promise to be an interesting laboratory for chiral interactions. This is an important supplement to investigations of $3 \mathrm{~N}$ scattering, where $3 \mathrm{~N}$ force effects have been identified mostly for higher energies [10].

\section{THE 3N AND 4N SYSTEMS}

A complete study of the $3 \mathrm{~N}$ system and the $4 \mathrm{~N}$ bound state can be found in Ref. [3]. Here we give a short summary of the results.

At NLO the Hamiltonian is completely determined by a fit to $2 \mathrm{~N}$ data, whereas the $3 \mathrm{NF}$ at NNLO involve two unknown LEC's, which were determined by a fit to the ${ }^{3} \mathrm{H} \mathrm{BE}$ and the doublet neutron-deuteron (nd) scattering length. Because the current interaction model does not include charge-independence breaking (CIB), we fitted to experimental values, which were corrected for this fact (see Ref. [3]). This was performed for two different cut-off values $\Lambda=500$ and $600 \mathrm{MeV}$. They are boundaries of a $\Lambda$ region, which ensures that physically interesting momenta are not affected by the cut-off and all LEC's remain natural at the same time. The resulting LEC's of the $3 \mathrm{NF}$ are $c_{D}=3.6, c_{E}=0.37$ and $c_{D}=1.8, c_{E}=-0.11$, respectively.

Comparing the differential elastic nd cross section at low energies, e.g. $3 \mathrm{MeV}$, for NLO and NNLO, one finds almost identical predictions. There is also only a very small cut-off dependence. For this observable and at this energy the chiral expansion seems to be converged. The nd cross section minimum at $65 \mathrm{MeV}$ gets a visible contribution from NNLO. For the $c_{D}$ and $c_{E}$ values obtained by the fit, the predictions at NNLO are in agreement with the data and the $\Lambda$ dependence is small. This indicates that the cross section is converged at this order. Interestingly, the agreement with the data vanishes, when changing to $c_{D}=-3.0$ for $\Lambda=500 \mathrm{MeV}$, while determining $c_{E}$ by a fit to the $3 \mathrm{~N}$ BE. This shows the sensitivity of the cross section minimum to the details of the $3 \mathrm{NF}$. It also indicates that the reduced contribution of the subleading $2 \pi$ exchange still 
leads to a consistent theory of the nuclear interaction. In contrast, the analyzing power $A_{y}$ for nd scattering at $3 \mathrm{MeV}$ changes, when increasing the order from NLO to NNLO. The prediction at NNLO does also not agree with the experiment. Surprisingly, for this observable, the chiral expansion is not converged.

Once the $3 \mathrm{NF}$ has been determined, the $4 \mathrm{~N}$ BE is a complete prediction at NNLO, because no $4 \mathrm{~N}$ force occurs at this order. We obtain $4 \mathrm{~N}$ BE's between $29.5 \mathrm{MeV}$ and $30.0 \mathrm{MeV}$ depending on $\Lambda$. This nicely agrees with the corrected experimental value of $29.8 \mathrm{MeV}$. The cut-off dependence is $500 \mathrm{keV}$ or $1.7 \%$ of the BE. However, calculations based on traditional forces have shown that the $3 \mathrm{~N}$ and $4 \mathrm{~N}$ BE are correlated [7]. Therefore, BE results for light nuclei are necessary to gain more confidence in the expansion for bound states and in the structure of the predicted $3 \mathrm{NF}$.

\section{THE ${ }^{6}$ Li SYSTEM}

${ }^{6} \mathrm{Li}$ is a first example. Calculations based on traditional forces have shown that the BE of the $1^{+}$ground state and the excitation energy of the $3^{+}$first excited state depend on the $3 \mathrm{NF}$ model, even if the $3 \mathrm{~N} \mathrm{BE}$ is correctly predicted [8,9]. The nucleus promises to be a good laboratory to probe the chiral $3 \mathrm{NF}$.

The chiral forces are non-local because of their regularization. This implies that only a very limited number of methods exist to solve the $A=6$ nuclear bound state problem. Here we employ the no-core shell model (NCSM) [11,12]. For NCSM, all nucleons remain active and no inert core is assumed. Therefore, a systematic procedure exits, which enables to calculate effective interactions from bare $2 \mathrm{~N}$ and $3 \mathrm{~N}$ forces (see [9]). The effective interactions speed up convergence sufficiently to obtain realistic results. We were able to perform calculations up to shell excitations of $N=6$, which means model space sizes for ${ }^{6} \mathrm{Li}$ of $d=197822$. The bare $3 \mathrm{NF}$ 's require the usage of effective three-body forces, which were obtained from solutions of $3 \mathrm{~N}$ cluster equations. All calculations were performed for an harmonic oscillator frequency of $13 \mathrm{MeV}$.

Fig. 1 shows the convergence of the BE's for different chiral interactions. With the exception of NLO for $\Lambda=500 \mathrm{MeV}$, the figure indicates convergence of the BE's to a level, which will be sufficient to study the $\Lambda$ dependence of the results. We observed the same behavior for the excitation energy, where the NLO result for $\Lambda=500 \mathrm{MeV}$ must also be taken with care.

For the BE's the $\Lambda$ dependence is shown in Fig. 2. To give an idea of the quality of the description, we also show the experimental $\mathrm{BE}$ and an estimated correction for the missing CIB, which we obtained by simply scaling the known correction in ${ }^{3} \mathrm{H}$. The Coulomb force was included into the calculations. One finds that the experimental value is within the bands. Going from NLO to NNLO one observes a reduction of the $\Lambda$ dependence. At NNLO it is $1.8 \mathrm{MeV}$ or $5.7 \%$ of the BE. Note that the $\Lambda$ dependence

of the NLO results might be larger than indicated, because of the slow convergence for NLO at $\Lambda=500 \mathrm{MeV}$. The same holds true for the excitation energy shown in Fig. 3. At NNLO, the $\Lambda$ dependence remains at $170 \mathrm{keV}$ or $7.6 \%$ of the excitation energy. Also here, the experimental value is within the band. The prediction for the excitation going from NLO to NNLO changes visibly, showing the importance of NNLO terms. For this model, where the subleading $2 \pi$ exchange is reduced in strength, the description of the 
experimental value improves. For a final assessment, the effects of this reduction have to be understood in more detail. The application of chiral interactions based on a new regularization scheme will be very important in this respect [4]. The size of the bands indicate that higher orders might be necessary to predict these observables on the $1 \%$ level.

Finally, we would like to use the new Idaho N3LO interaction [13] in combination with the NNLO $3 \mathrm{NF}$ to investigate the sensitivity of the ${ }^{6} \mathrm{Li}$ system to the choice of $c_{D}$ and $c_{E}$. We determined two different sets of $c_{D}$ and $c_{E}$, which describe by construction the experimental ${ }^{3} \mathrm{H}$ and ${ }^{4} \mathrm{He} \mathrm{BE}$ 's for $\Lambda=500 \mathrm{MeV}$. The two sets are $c_{D}=-1.11, c_{E}=-0.66$ $(3 \mathrm{NF}-\mathrm{A})$ and $c_{D}=8.14, c_{E}=-2.02(3 \mathrm{NF}-\mathrm{B})$, respectively. Fig. 4 shows the convergence of our results with respect to $N$ for the different cases, which is acceptable except for Idaho N3LO and 3NF-B. Nevertheless, we can observe a strong dependence of the BE on the chosen $3 \mathrm{NF}$; an observation, which we could also make for the excitation energies. These first results confirm the importance of the shorter-range terms of the $3 \mathrm{NF}$ for light nuclei.

\section{CONCLUSIONS AND OUTLOOK}

Chiral perturbation theory promises a much better understanding of nuclear forces. The structure of the $3 \mathrm{NF}$ will be an important aspect, because it is not well understood phenomenologically and because it allows to study the subleading contributions, which is crucial to confirm the approach. In this respect it will be very interesting to apply forces based on different regularization schemes [4] to FN systems. New developments show that CIB and charge-symmetry breaking can be included on the same footing. Then it will be

very interesting to extend the calculations to neutron rich nuclei and explicitly probe the isospin dependence of the 3NF's.

Improvements in the algorithms for NCSM are currently developed. They will allow for larger spaces and further remove the uncertainties of $A>4$ calculations.

This work was supported in part by grants from the U.S. National Science Foundation, the U.S. Department of Energy and the Deutsche Forschungsgemeinschaft. The numerical calculations were performed on the IBM SP at NERSC in Berkley and the Cray complex of the NIC in Jülich, Germany.

\section{REFERENCES}

1. E. Epelbaum, H. Kamada, A. Nogga, H. Witała, W. Glöckle, U.-G. Meißner, Phys. Rev. Lett. 86 (2001) 4787.

2. E. Epelbaum, A. Nogga, W. Glöckle, H. Kamada, U.-G. Meißner, H. Witała, Eur. Phys. J. A 15 (2002) 543.

3. E. Epelbaum, A. Nogga, W. Glöckle, H. Kamada, U.-G. Meißner, H. Witała, Phys. Rev. C 66 (2002) 064001.

4. E. Epelbaum, et al., contribution to this conference.

5. E. Epelbaum, W. Glöckle, U.-G. Meißner, Nucl. Phys. A671 (2000) 295.

6. U. van Kolck, Phys. Rev. C 49 (1994) 2932.

7. A. Nogga, H. Kamada, W. Glöckle, B. R. Barrett, Phys. Rev. C 65 (2002) 054003. 


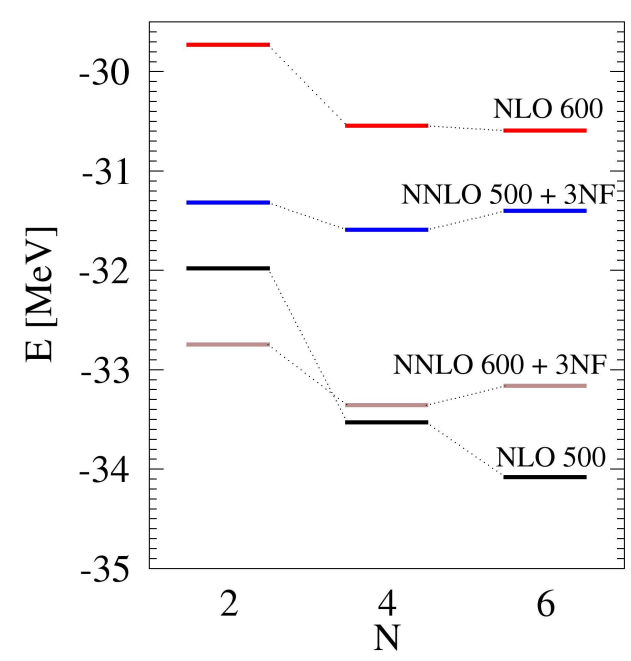

Figure 1. Binding energies of ${ }^{6} \mathrm{Li}$ for different chiral interactions. The NCSM model space sizes were truncated by the maximal excitation to $N=2,4$ or 6 .

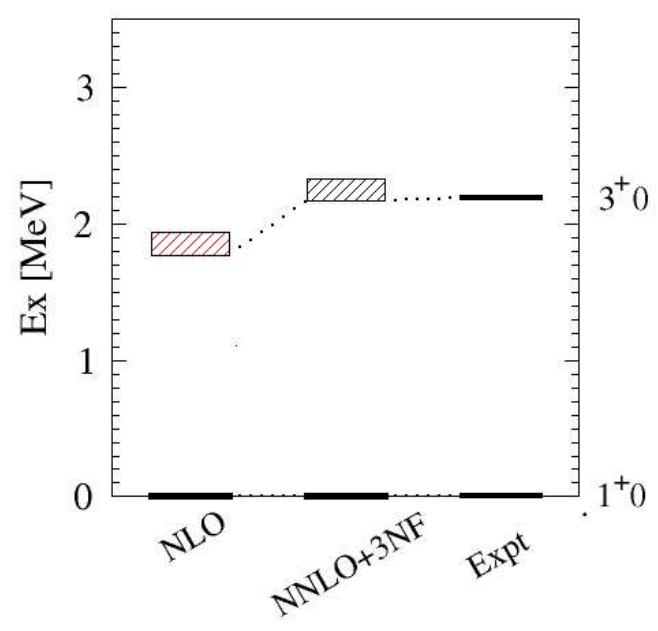

Figure 3. Same as Fig. 2 for the excitation energy the $3^{+}$state.

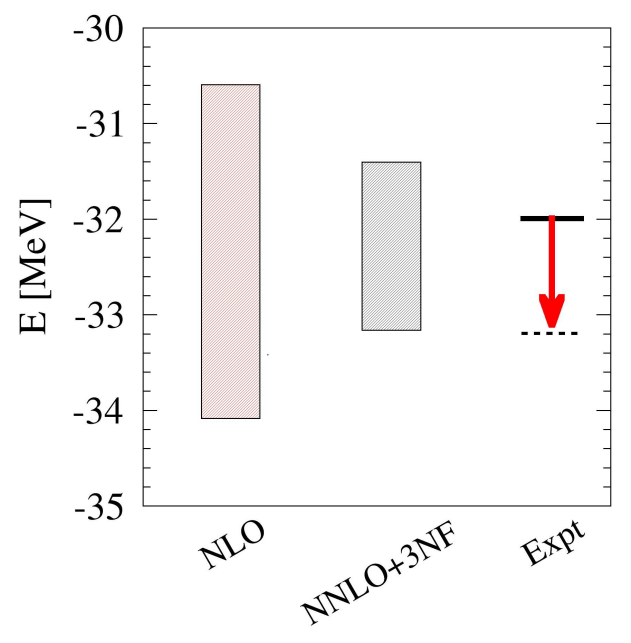

5

Figure 2. Binding energies of ${ }^{6} \mathrm{Li}$ for $\mathrm{NLO}$ and NNLO compared to the experimental value. The arrow indicates a rough estimate of CIB. The bands are given by variations of $\Lambda$ between 500 and $600 \mathrm{MeV}$.

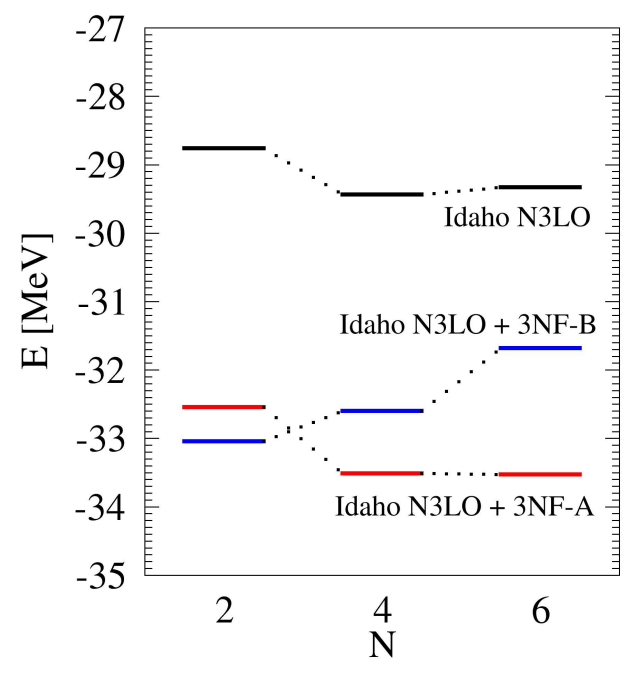

Figure 4. Same as Fig. 1 for different combinations with Idaho N3LO.

8. S. C. Pieper, V. R. Pandharipande, R. B. Wiringa, J. Carlson, Phys. Rev. C 64 (2001) 014001.

9. P. Navrátil, W. E. Ormand Nucl-th/0305090.

10. H. Witała, W. Glöckle, J. Golak, A. Nogga, H. Kamada, R. Skibiński, J. KurośŻołnierczuk, Phys. Rev. C 63 (2001) 024007.

11. P. Navrátil, J. P. Vary and B. R. Barrett, Phys. Rev. C 62 (2000) 054311.

12. P. Navrátil, W. E. Ormand, Phys. Rev. Lett. 88 (2002) 152502.

13. D. R. Entem, R. Machleidt, nucl-th/0304018. 
This work was performed under the auspices of the U.S. Department of Energy by University of California, Lawrence Livermore National Laboratory under Contract W-7405-Eng-48. 